I N S T I T U T O

DE

M E D I C I N A

T R O P I C A L

$\mathrm{DE}$

S ÃO PAULO

JOURNAL OF THE SÃO PAULO INSTITUTE OF TROPICAL MEDICINE

${ }^{1}$ Universidade Federal do Espírito Santo, Hospital Universitário Cassiano Antonio Moraes, Vitória, Espírito Santo, Brazil

'Universidade de São Paulo, Faculdade de Medicina, São Paulo, São Paulo, Brazil

*Those authors contributed equally to this study.

Correspondence to: Weverton Machado Luchi

Universidade Federal do Espírito Santo, Hospital Universitário Cassiano Antonio Moraes, Divisão de Nefrologia, Departamento de Clínica Médica, Av. Mal. Campos, 1355, Santa Cecília, CEP 29043-260, Vitória, ES, Brazil

E-mail: wmluchi@ hotmail.com

Received: 29 September 2021

Accepted: 3 January 2022

\section{Tenofovir-induced renal and bone toxicity: report of two cases and literature review}

\author{
Carlos Eduardo Andrade Fioroti ${ }^{1^{*}}$, Jesiree Iglésias Quadros Distenhreft ${ }^{1 *}$, \\ Bruna Bastos Paulino', Kamilla Lacchine', Danilo Rodrigues Ramos', \\ Antonio Carlos Seguro², Weverton Machado Luchi ${ }^{1}$
}

\section{ABSTRACT}

Tenofovir Disoproxil Fumarate (TDF) is one of the drugs in the initial first-line antiretroviral regimen for the treatment of hepatitis B and HIV infections. Despite its effectiveness and few adverse effects, it is related to renal and bone toxicity. We described two cases of HIVpositive middle-aged women who had been using TDF for two and four years (cases 1 and 2 , respectively) and were admitted to the emergency room. Case 1 presented with metabolic ileum and diffuse bone pain while case 2 presented with bilateral coxo-femoral pain after a fall from standing height. Both cases had similar laboratory tests: hyperchloremic metabolic acidosis, hypophosphatemia, hypokalemia, hypouricemia and elevated plasma creatinine. In urinary exams, there was evidence of renal loss of electrolytes, justifying the serum alterations, in addition to glucosuria and proteinuria. The bone pain investigation identified bone fractures and reduced bone mineral density, together with increased levels of parathyroid hormone, alkaline phosphatase and vitamin D deficiency. These two cases illustrate the spectrum of adverse renal and bone effects associated with TDF use. TDF was discontinued and treatment was focused on correcting the electrolyte disturbances and acidosis, in addition to controlling the bone disease through vitamin $\mathrm{D}$ and calcium supplementation. The renal changes found in both cases characterized the Fanconi's syndrome, and occurred due to TDF toxicity to proximal tubule cells mitochondria. Bone toxicity occurred due to direct interference of TDF in bone homeostasis, in addition to vitamin D deficiency and phosphaturia resulting from tubulopathy. During the follow-up, both cases evolved with chronic kidney disease and in one of them, the Fanconi's syndrome did not revert. We emphasize the need to monitor markers of bone metabolism and glomerular and tubular functions in patients using TDF.

KEYWORDS: Tenofovir Disoproxil Fumarate. Anti-retroviral agents. HIV. Kidney. Nephrotoxicity. Bone.

\section{INTRODUCTION}

Tenofovir Disoproxil Fumarate (TDF) is one of the drugs in the initial first-line antiretroviral regimen for the treatment of hepatitis B and HIV infections. It belongs to the class of nucleotide reverse transcriptase inhibitors and despite its significant efficacy, good tolerability and low incidence of metabolic adverse effects, TDF is related to more than half of the cases of tubulopathies caused by the antiretroviral therapy in HIV-infected patients. In addition to nephrotoxicity, another common adverse effect is bone toxicity, caused by direct and indirect effects of the drug and characterized by osteopenia/osteoporosis, osteomalacia and bone fractures ${ }^{1}$. Next, we report two clinical cases in HIV-infected patients to illustrate the broad 
spectrum of clinical and laboratory manifestations caused by tenofovir toxicity to kidneys and bones, emphasizing the pathophysiology of these alterations.

\section{CASE REPORTS}

Case 1: A 55-year-old woman, HIV-positive for thirteen years, using zidovudine, lamivudine and TDF, was admitted to the emergency room because of a 1-week progressive abdominal pain and distension, associated with interruption of flatus and feces elimination. In addition, she reported diffuse bone pain, more intense in the hip, that had started one year before, but worsened during the last month. The acute abdominal pain protocol ruled out obstructive pathologies, and the analysis of laboratory tests suggested the diagnosis of metabolic ileus (Table 1). Further investigation of the patient's bone-articular system revealed the presence of fractures of the right ischiopubic ramus and of the second and fifth costal arches on the left side, and osteonecrosis of the right femoral head (Figures 1A and 1B).

Case 2: A 62-year-old woman, HIV-positive for four years, using lamivudine, efavirenz and TDF, was admitted to the emergency room due to bilateral coxo-femoral pain after a fall from standing height. The investigation revealed fractures with partial consolidation of the right femoral neck and left femoral sub-trochanteric region and old fractures in the ischiopubic ramus and right pubis (Figures $1 \mathrm{C}$ and 1D). She underwent surgical correction with subsequent hospital discharge. However, after one month, she was readmitted with progressive muscle weakness, diffuse paresthesia and vomiting.

Patients 1 and 2 had been using an antiretroviral therapy (ART) regimen containing TDF for two and four years,

Table 1 - Laboratory tests referring to clinical cases 1 and 2.

\begin{tabular}{|c|c|c|c|c|c|c|c|c|c|}
\hline \multirow{2}{*}{ Exams } & \multicolumn{4}{|c|}{ Case 1} & \multirow{2}{*}{$\begin{array}{l}\text { Reference } \\
\text { values }\end{array}$} & \multicolumn{4}{|c|}{ Case 2} \\
\hline & Admission & 2 months & 12 months & 36 months & & Admission & 2 months & 12 months & 36 months \\
\hline & & & & & Blood & & & & \\
\hline Creatinine (mg/dL) & 2.41 & 1.55 & 1.31 & 1.1 & $0.5-0.9$ & 2.2 & 1.41 & 1.5 & 1.42 \\
\hline Urea (mg/dL) & 25 & 44 & 45 & 27 & $16-40$ & 53 & - & 78 & 52 \\
\hline $\mathrm{pH}$ & 7.15 & 7.36 & 7.41 & - & $7.35-7.45$ & 7.25 & 7.32 & 7.3 & 7.27 \\
\hline Bicarbonate (mmol/L) & 11 & 24.5 & 24.2 & - & $22-26$ & 10.6 & 22.7 & 19.4 & 15.4 \\
\hline Anion Gap & 18 & 11.5 & 13.8 & - & $10-12$ & 10.4 & - & - & - \\
\hline Chloride (mEq/L) & 114 & 106 & 103 & 106 & $98-109$ & 118 & - & 106 & 106 \\
\hline Sodium (mEq/L) & 143 & 142 & 141 & 142 & $135-145$ & 139 & - & 140 & 141 \\
\hline Potassium (mEq/L) & 2.3 & 4.34 & 4.4 & 4.4 & $3.5-5.1$ & 1.7 & 3.7 & 3.8 & 4.0 \\
\hline Magnesium (mEq/L) & - & 2.22 & 2.46 & 2.3 & $1.7-2.5$ & 1.5 & 1.97 & - & - \\
\hline Calcium (mg/dL) & - & 8.9 & 10.4 & 9.5 & $8.6-10$ & 8.9 & 9.8 & 9.2 & 9.3 \\
\hline Phosphorus (mg/dL) & 2.1 & - & 3.74 & 4.1 & $2.5-4.5$ & 1.7 & 2.32 & 2.4 & - \\
\hline Uric acid (mg/dL) & 2 & 1.98 & - & 2.5 & $2.6-6.7$ & 1.2 & - & - & - \\
\hline Glucose (mg/dL) & 92 & - & 104 & - & $60-110$ & - & - & 93 & 93 \\
\hline Parathormone (pg/mL) & 98 & 35 & 69 & 67 & $15-68.3$ & 606 & 55.7 & 197 & 156 \\
\hline Alkaline Phosphatase (U/L) & 758 & 1,210 & 154 & 163 & $68-240$ & 1,032 & 978 & 1,032 & - \\
\hline \multirow[t]{2}{*}{ 25-OH Vitamin D (ng/mL) } & 17 & - & 32 & 30 & $>30$ & 9 & 46 & 22 & 29 \\
\hline & \multicolumn{9}{|c|}{ Urinalysis } \\
\hline $\mathrm{pH}$ & 7.0 & 7.0 & - & 5.0 & $5.5-6.5$ & 8.0 & - & 6.0 & 7.0 \\
\hline Density & 1.005 & 1.020 & - & 1.009 & $1.015-1.025$ & 1.010 & - & 1.019 & 1.018 \\
\hline Albumin (mg/dL) & ++ & 75 & absent & absent & $<15$ & + & 100 & 100 & 150 \\
\hline Glucose (mg/dL) & ++ & 100 & absent & absent & $<30$ & +++ & 300 & 300 & 300 \\
\hline Anion Gap & 14 & - & - & - & & 38 & - & - & - \\
\hline \multirow[t]{2}{*}{$\begin{array}{l}\text { Albumin-creatinine ratio } \\
(\mathrm{mg} / \mathrm{g})\end{array}$} & - & - & - & - & - & - & 3,800 & - & - \\
\hline & \multicolumn{9}{|c|}{ 24-Hour urinalysis } \\
\hline 24-hour proteinuria (mg) & 1,680 & 74 & - & 46 & $<150$ & - & - & - & - \\
\hline 24-hour glucosuria (g) & 11 & absent & - & - & $<0.5$ & - & - & - & - \\
\hline FE* Phosphate (\%) & 56 & - & - & - & $15-20$ & 52 & 49 & - & - \\
\hline FE* Potassium (\%) & 35 & 32 & - & - & $4-16$ & 51 & 25 & - & - \\
\hline FE* Uric acid (\%) & 31 & 42 & - & 5 & $<10$ & - & - & - & - \\
\hline $\mathrm{TmP} \mathrm{GFR}^{* *}(\mathrm{mg} / \mathrm{dL})$ & 0.22 & - & - & - & $2.2-3.6$ & 0.36 & - & - & - \\
\hline
\end{tabular}

${ }^{\star} F E=$ Fractional Excretion; ${ }^{* *} T M P /$ GFR $=$ Transport Maximum for Phosphate reabsorption/Glomerular Filtration Rate; ${ }^{* * *}$ FE of phosphate (\%) calculator $=[($ PO4(urine) $/$ PO4(serum) $/($ Creatinine(urine) $/$ Creatinine $($ serum $)] \times 100$. 

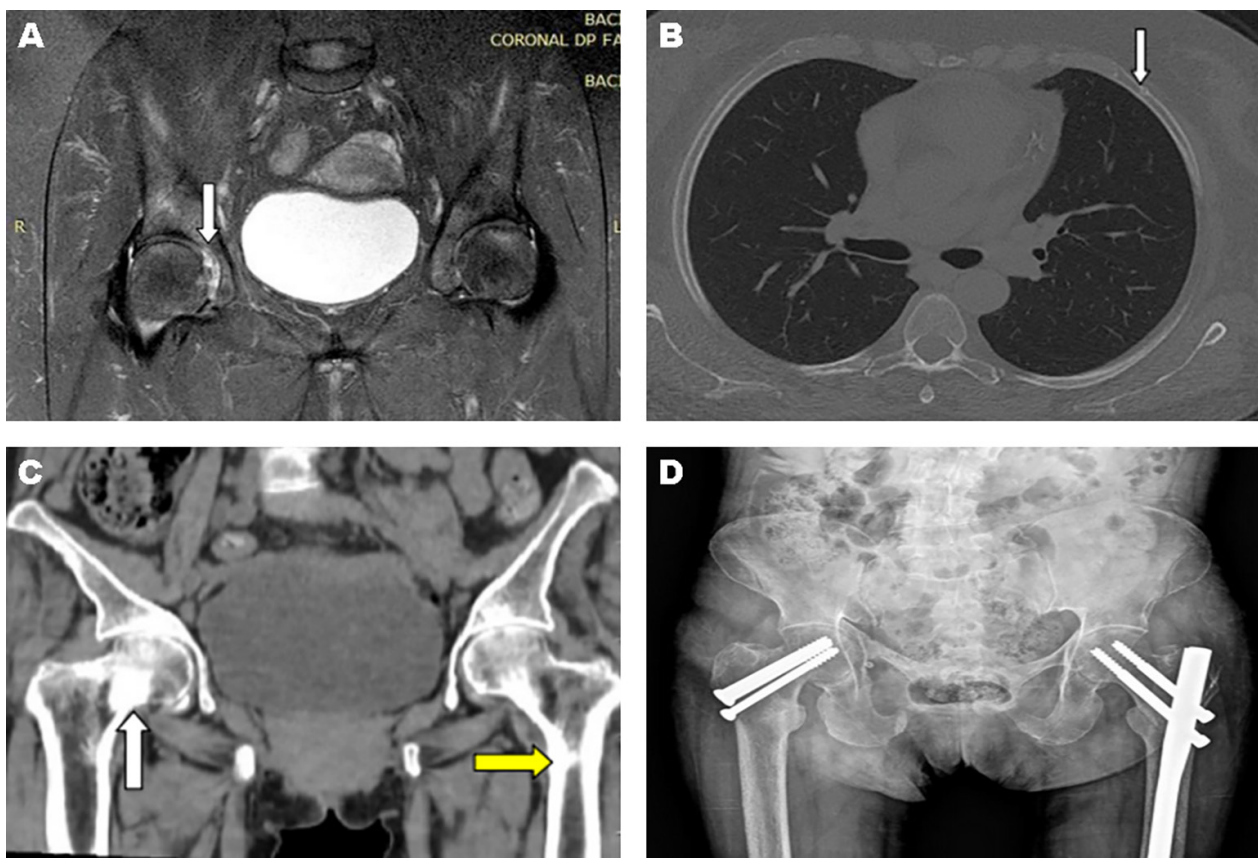

Figure 1 - Image exams referring to clinical cases 1 (A and $B)$ and $2(C$ and $D):(A) M R I$ of the hip with osteonecrosis of the right femoral head (white arrow); (B) CT of the chest showing an incomplete fracture of the fifth left costal arch (white arrow); (C) CT of the pelvis showing fractures with partial consolidation of the right femoral neck (white arrow) and left subtrochanteric (yellow arrow);

(D) Postoperative control - pelvis X-ray.

respectively. Although the patients were admitted with different clinical conditions (acute abdomen vs. femur fracture), both patients had the same pattern of laboratory alterations, as shown in Table 1: hyperchloremic metabolic acidosis, hypophosphatemia, hypokalemia, hypouricemia and elevation of plasma creatinine. Urine analysis showed an "alkaline" urinary $\mathrm{pH}$, glycosuria and proteinuria. Increased fractional excretion (FE) of phosphate and potassium and reduction in maximum phosphorus transport/ glomerular filtration rate (TmP/GFR) indicated renal loss of these electrolytes. In addition, parathyroid hormone $(\mathrm{PTH})$ and alkaline phosphatase were increased, while 25-hydroxyvitamin D (calcidiol) was reduced. Dual-energy X-ray absorptiometry (DXA) T-score of the femoral neck was -2.7 in case 1 and -4.5 in the lumbar spine of case 2 .

\section{Diagnoses}

Tenofovir-related renal and bone toxicity: Acute Kidney Injury (AKI), Fanconi's syndrome (FS), vitamin D deficiency, secondary hyperparathyroidism (SHPT), bone mineral density (BMD) reduction and bone fractures.

\section{Follow-up}

TDF was discontinued and the ART regimen was switched, in case 1 to abacavir, lamivudine and fosamprenavir/ritonavir, and in case 2 to abacavir, lamivudine, darunavir/ritonavir. In both cases, the initial treatment was based on oral replacements of potassium, bicarbonate, vitamin $\mathrm{D}$ (cholecalciferol) and calcium. Later (6-12 months after starting treatment), after controlling acid-base and electrolyte disturbances and SHPT, a bisphosphonate $(70 \mathrm{mg} /$ week of alendronate sodium) was introduced for osteoporosis. In case 1, the metabolic ileum improved after correction of electrolytes and acid-base disturbances. During the outpatient follow-up, complete resolution of hypokalemia, hypophosphatemia, hypouricemia and acidosis, as well as SHPT and urinary alterations (proteinuria and glycosuria) were observed, allowing the suspension of oral replacements after six months. In addition, DXA control showed a T-score of -0.9 in the femoral neck after 18 months. However, GFR has only partially improved, evolving to stage $3 \mathrm{~A}$ of chronic kidney disease (CKD) (CKD-EPI of $56 \mathrm{~mL} / \mathrm{min} / 1.73 \mathrm{~m}^{2}$ ) after 32 months (Table 1). In case 2, during the first 12 months of treatment, it was necessary to maintain the oral replacements initially introduced. Then, the patient was lost to follow-up, returning 24 months later. New tests showed persistence of proteinuria, glycosuria, metabolic acidosis, SHPT and stage 3B CKD (CKD-EPI $42 \mathrm{~mL} / \mathrm{min} / 1.73 \mathrm{~m}^{2}$ ), suggesting irreversibility of FS (Table 1).

\section{DISCUSSION}

These two clinical cases illustrate the broad spectrum 
of adverse effects of TDF involving kidneys and bones, as we will review below. Unlike the kidney damage associated with HIV, which has a predilection for the glomerulus, the main target of renal aggression by antiretroviral drugs is the tubule-interstitial compartment. In two large retrospective studies involving kidney biopsies from HIV-positive patients, one evaluating 222 and the other 437 patients, TDF nephrotoxicity accounted for $13-16 \%$ of all histological findings, and 49-59\% of cases with tubule-interstitial diseases ${ }^{2,3}$. Among drug-related tubuleinterstitial nephrotoxicity, TDF was the main offending agent, accounting for $70 \%$ of the cases ${ }^{2}$. In general, TDF renal toxicity increases with time of drug use, becoming more evident after the first year. Additional risk factors include age ( $>50$ years), low body weight $(<60 \mathrm{~kg})$, use of other nephrotoxic drugs, male gender, reduced glomerular filtration rate (GFR), comorbidities (HIV-Hepatitis C coinfection, diabetes, hypertension), advanced HIV infection (low CD4 lymphocyte counts, AIDS) and vitamin D deficiency ${ }^{1,4,5}$.

Although the pathological mechanisms are not fully elucidated, the renal damage generated by TDF seems to be related to its toxicity to proximal tubular cells mitochondrial DNA through inhibition of the gamma polymerase enzyme $e^{4,6}$. Consequently, there will be dysfunction in the mitochondrial oxidative phosphorylation process with generation of oxidative stress and low ATP levels. These changes will interfere with the expression of transport proteins on the luminal surface of tubular cells and their calcitriol (1,25-dihydroxyvitamin $\mathrm{D})$ production. Furthermore, mitochondrial damage can also stimulate apoptosis of tubular cells by activating the caspase pathway (Figure 2 Ia). Additionally, in rats, TDF nephrotoxicity was accompanied by downregulation of endothelial nitric oxide synthase protein concentration and severe renal vasoconstriction ${ }^{7}$. Histological findings in renal biopsies are characterized by acute tubular necrosis associated with eosinophilic intracytoplasmic inclusions, which correspond to dysmorphic and giant mitochondria ${ }^{6}$.

About 76-90\% of cases of TDF-induced kidney damage displayed proximal tubular dysfunction, clinically manifested by AKI, proteinuria (predominantly tubular proteinuria) and urinary active sediment. On the other hand, CKD was among the indications for kidney biopsy in about $24 \%$ of cases $^{2,3}$. In general, there is an impairment in the reabsorption of glucose, phosphate, uric acid, amino acids, tubular proteins (as $\beta 2$-microglobulin) and bicarbonate in the proximal tubule. Renal loss and increased urinary excretion of all these substances will not always be present simultaneously, but when it happens
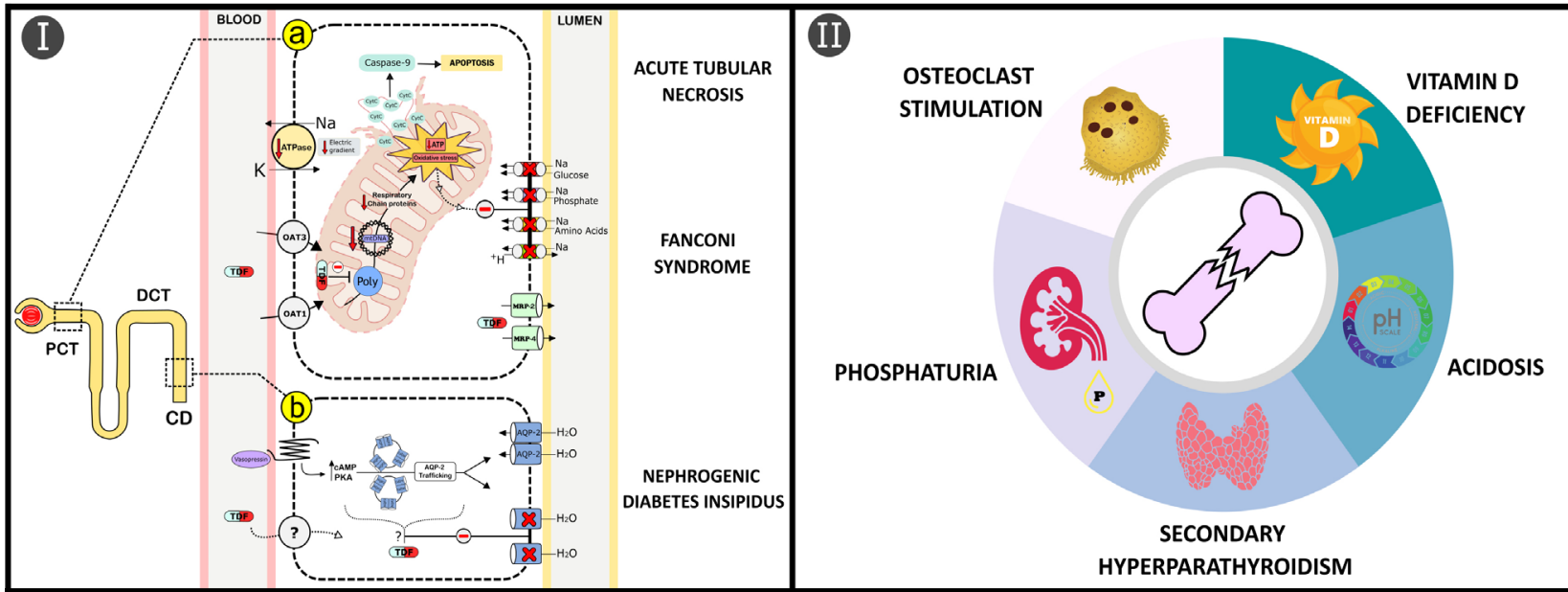

Figure 2 - Potential pathogenic mechanisms of TDF-induced bone and renal toxicity: (la) After entering the proximal convoluted tubule (PCT) epithelial cells by OAT1/OAT3 transporters, TDF triggers functional and structural abnormalities in mitochondria through the inhibition of DNA polymerase $\gamma$ enzyme (Pol $\gamma$ ), compromising mitochondrial DNA (mtDNA) synthesis and production of respiratory chain proteins. In this scenario, there will be a reduction in the supply of ATP to the cell, in addition to the generation of oxidative stress, leading to decreased basolateral $\mathrm{Na} / \mathrm{K}$-ATPase activity, interfering with the trafficking and endosomal recycling of apical membrane transporters in polarized epithelial cells. Furthermore, the release of proteins from mitochondria to the cytosol, including cytochrome c (CytC), will stimulate apoptosis via caspase-9 pathway and damage to the cellular DNA; (Ib) In the distal nephron, collecting duct (CD) cells can also be targeted for damage by TDF through reduced expression of aquaporin-2 (AQP-2) channels on the luminal surface. However, since there is no expression of OAT1/OAT3 in human CD cells, the mechanisms of entry into cells, as well as the reduction in the expression of AQP-2 in the luminal membrane are still unknown. The spectrum of kidney damage includes acute tubular necrosis, Fanconi's syndrome and nephrogenic diabetes insipidus; (II) TDF interferes directly with bone homeostasis, stimulating osteoclastic differentiation, and indirectly through PCT epithelial cells damage, reducing the production of calcitriol that is responsible for the development of secondary hyperparathyroidism, and inducing phosphaturia and systemic acidosis by bicarbonaturia. 
(27-40\% of cases), the FS is characterized ${ }^{2-4}$. The main transport affected is involved in phosphorus reabsorption, and as a consequence, hypophosphatemia is a frequent finding. Another manifestation described in case series by Zaidan et al. ${ }^{2}$ was nephrogenic diabetes insipidus, present in approximately $7 \%$ of patients with tubule-interstitial damage attributed to TDF. An experimental study showed that TDF reduces the expression of aquaporin-2 channels in collecting duct cells (Figure $2 \mathrm{Ib})^{7}$.

Clearance of TDF occurs through a combination of glomerular filtration and tubular secretion. About 20-30\% of the drug is actively transported across the basolateral membrane of proximal tubule cells by organic anion transporters, in particular OAT- 1 and to a lesser extent OAT-3. Then, the secretion into the tubular lumen occurs via energy-dependent pumps, the so-called multidrug resistance proteins (MRP-2 and MRP-4) (Figure 2 Ia) ${ }^{4}$. In this scenario, the increase in the intracellular concentration of TDF, aggravating the tubular lesion, can occur in the following situations: drop in GFR, resulting in increased drug elimination by the secretory pathway; or by the use of drugs that increase the activity of OAT-1 and OAT-2 transports and/or reduce MRP-2 and MRP-4. As an example, ritonavir-booster protease inhibitors (PI/RTV) compete with TDF for MRP-2 transport, increasing the risk of kidney injury due to TDF 3.7 times $^{8}$. Studies on pharmacokinetic have shown that boosted protease inhibitors regimens (bPIs) significantly increase the area under de curve of TDF plasma concentrations by $25-37 \%$. Non-steroidal anti-inflammatory drugs, acyclovir and ganciclovir may also increase TDF nephrotoxicity by inhibiting MRP-4. Furthermore, a single nucleotide polymorphism in the ABCC2 gene (1249 $\mathrm{G} \rightarrow \mathrm{A}$ ), responsible for encoding MRP-2, is associated with a 5-6 times greater risk of tubular toxicity and progression to $\mathrm{FS}^{6}$. In contrast, drugs such as probenecid, an uricosuric that inhibits OAT-1, has been shown to be potentially effective in reducing TDF nephrotoxicity ${ }^{4,10}$.

Tenofovir Alafenamide (TAF), a new prodrug of tenofovir, has recently emerged as an alternative to TDF. After oral administration, whereas TDF is hydrolyzed by intestinal and plasma esterases to tenofovir, TAF is predominantly metabolized intracellularly by cathepsin A to tenofovir. Thus, the pharmacokinetics of TAF allowed a reduction of about $91 \%$ in plasma concentrations of the active metabolite of tenofovir when compared to TDF, reducing kidneys and bones exposure to the drug. On the other hand, TAF increased intracellular concentrations by 6.5 times, allowing the ingestion of lower doses of the medication ( $25 \mathrm{mg}$ of TAF is bioequivalent to $300 \mathrm{mg}$ of TDF in terms of plasma levels of tenofovir ${ }^{9,11}$. In phase 3 studies, patients with HIV and chronic hepatitis B who started treatment with TAF-containing regimens had significantly lower decrease in GFR, less proteinuria and less reduction in BMD in comparison with those receiving TDF-containing regimens. In addition, patients on TDF who migrated to TAF had increased $\mathrm{BMD}^{11}$. These findings were also reinforced by a meta-analysis of randomized controlled trials conducted by Tao et al. ${ }^{12}$. However, the supposed security benefits of TAF over TDF seem to be overestimated, since the adverse effects are more apparent when TDF is administrated as part of bPIs. In a metaanalysis of randomized controlled trials conducted by Hill et al. ${ }^{9}$, recently updated, TAF was compared with TDF in boosted or unboosted subgroups for treatment of HIV and chronic hepatitis $\mathrm{B}^{13}$. This study demonstrated that, in comparison with TAF, TDF was associated with higher risks of bone and renal adverse events only when boosted with RTV or cobicistat (COBI). By contrast, when RTV and COBI were not used (unboosted subgroups), there were no differences between TAF and TDF for HIV RNA suppression, clinical adverse events, discontinuation due to renal adverse events, bone fractures or discontinuation due to bone-related adverse events. In addition, a recent systematic review described by Fraga et al. ${ }^{14}$ has also emphasized that renal and bone toxicity of TDF appears to be a potential problem in HBV/HIV coinfected patients, although it is not clinically relevant in HBV infections only treated with a single drug therapy. It is speculated that the absence of TDF dose adjustment when combined with bPIs (TAF is reduced from $25 \mathrm{mg}$ to $10 \mathrm{mg}$ per day, but the TDF dose is maintained at $300 \mathrm{mg}$ per day) results in lower tolerability of TDF compared to TAF regimens ${ }^{13}$.

$\mathrm{HIV}$ infection is an isolated risk factor for reduced BMD in children and adults. Osteopenia and osteoporosis rates in HIV-positive populations range from $42-67 \%$ and $12-23 \%$, respectively, with a prevalence 6.7 and 3.7 times higher when compared to uninfected populations. The result is a risk of fracture $60 \%$ higher than the general population. In this scenario, the vast majority of ART regimens contribute to the reduction in BMD. Studies have shown a 2-6\% reduction in BMD in the first two years after ART initiation, and regimens containing TDF cause the greatest reductions in BMD when compared to others ${ }^{15,16}$.

Experimental studies have shown that TDF directly interferes with bone homeostasis through the reduction of extracellular adenosine levels, mediated by inhibition of ATP release from cells ${ }^{17}$. As a result, there will be stimulation of osteoclast differentiation and osteoblast inhibition, with increased bone resorption. In addition, TDF interferes with the binding of calcidiol with its carrier protein (DBP, vitamin D binding protein) reducing its 
availability for the production of 1.25-dihydroxyvitamin $\mathrm{D}$ (calcitriol) in the kidney, the biologically active form ${ }^{18}$. A summary of TDF-induced bone toxicity mechanisms is illustrated in Figure 2 II.

The indirect effects of TDF on bones are related to mitochondrial toxicity of proximal convoluted tubule renal cells, which may trigger the following changes: 1) urinary calcidiol loss due to the inability to reabsorb DBP and reduction of the mitochondrial 1-alpha-hydroxylase enzyme-mediated conversion of calcidiol to calcitriol; 2) phosphaturia consequent to reduced expression of the $\mathrm{NaPi}$-IIa cotransport; 3) metabolic acidosis secondary to bicarbonate reabsorption deficiency (Figure 2 Ia) ${ }^{4,7,19}$. Reducing calcitriol will also reduce the absorption of calcium and phosphorus in the intestine and stimulate the development of SHPT. Acidemia and SHPT stimulate the increase in osteoclastic activity/bone turnover induced by TDF itself. Furthermore, phosphate wasting may be associated with osteomalacia, characterized by impaired bone mineralization. As DXA scanning may not differentiate between osteoporosis and osteomalacia, the presence of osteomalacia may go unnoticed. However, clinical (bone pain and fractures) and laboratory (hypophosphatemia, vitamin D deficiency and elevated serum alkaline phosphatase level as a compensatory increase in osteoblast activity) findings may suggest the presence of this medical condition, as observed in our patients ${ }^{19,20}$.

The treatment of toxicity induced by TDF should be directed to the changes found in the clinical presentation. The focus will be on the correction of electrolyte disturbances and metabolic acidosis associated with tubulopathy, in addition to the control of SHPT and reduced bone mass with vitamin $\mathrm{D}$ and calcium supplementation. Replacement of TDF for a non-tenofovir regimen or TAF is suggested by expert opinion if: GFR is $\leq 60 \mathrm{~mL} / \mathrm{min}$, urine protein/creatinine ration (UP/C) $>50 \mathrm{mg} / \mathrm{mmol}$, glucosuria is present in non-diabetics, confirmed hypophosphatemia of renal origin and osteopenia/ osteoporosis in the presence of increased urine phosphate leak. In addition, replacement should also be considered in the following situations: GFR $>60 \mathrm{~mL} / \mathrm{min}$, but decrease in GFR by $5 \mathrm{~mL} / \mathrm{min}$ per year for at least three consecutive years or confirmed $25 \%$ GFR decline from baseline, UP/C $15-50 \mathrm{mg} / \mathrm{mmol}$, presence of comorbidities with a high risk of CKD (i.e. diabetes and hypertension), body weight $<60 \mathrm{~kg}$ or use of a PI/RTV as a third therapeutic agent ${ }^{1}$. After drug withdrawal, there is a tendency for the recovery of renal functions, which can vary from days to months, a period in which oral replacements of potassium, sodium bicarbonate, calcium, phosphate and cholecalciferol may be necessary. However, progression to
CKD and tubular alterations may remain in up to $60-70 \%$ of patients ${ }^{2,6}$. In cases in which FS becomes persistent, calcitriol supplementation may be necessary due to the kidney's inability to produce active vitamin D. Treatment of osteoporosis, when present, may include anti-resorptive drugs such as bisphosphonates or denosumab. However, it is important to emphasize that the use of these drugs can aggravate hypophosphatemia and induce fractures in patients with FS/osteomalacia, and therefore, should only be considered when these conditions are controlled ${ }^{20}$.

\section{CONCLUSION}

Monitoring glomerular and tubular functions along with mineral and bone metabolism markers is crucial in HIV-positive patients using TDF. Serum dosages of creatinine, phosphorus, potassium, bicarbonate, PTH, 25-hydroxyvitamin D and alkaline phosphatase, as well as a urine sample (first morning) for the evaluation of UP/C, phosphaturia (quantified as FE of phosphate) and urinalysis are recommended annually/biannually in outpatients follow-up, in addition to DXA performed every two years. ${ }^{1}$ This monitoring should be more rigorous in patients with risk factors for nephrotoxicity and in those who have a history of fragility fractures, osteopenia/osteoporosis or a high FRAX score. In addition, it is important to pay attention to the association of other drugs that may aggravate TDF toxicity, especially when TDF is used in combination with bPIs.

\section{AUTHORS' CONTRIBUTIONS}

CEAF and WML were responsible for the diagnosis and follow-up of the patients. Material preparation and data collection were performed by CEAF, JIQD, BBP, KL, DRR and WML. The first draft of the manuscript was written by CEAF, JIQD and WML. ACS commented on previous versions of the manuscript and contributed to the preparation of the final version. All authors read and approved the final manuscript.

\section{CONFLICT OF INTERESTS}

The authors declared no potential conflict of interests with respect to the research, authorship, and/or publication of this article.

\section{FUNDING}

The authors received no financial support for the research, authorship, and/or publication of this article. 


\section{REFERENCES}

1. Ryom L, Cotter A, De Miguel R, Béguelin C, Podlekareva D, Arribas JR, et al. 2019 update of the European AIDS Clinical Society Guidelines for treatment of people living with HIV version 10.0. HIV Med. 2020;21:617-24.

2. Zaidan M, Lescure FX, Brochériou I, Dettwiler S, Guiard-Schmid JB, Pacanowski J, et al. Tubulointerstitial nephropathies in HIV-infected patients over the past 15 years: a clinicopathological study. Clin J Am Soc Nephrol. 2013;8:930-8.

3. Kudose S, Santoriello D, Bomback AS, Stokes MB, Batal I, Markowitz GS, et al. The spectrum of kidney biopsy findings in HIV-infected patients in the modern era. Kidney Int 2020;97:1006-16.

4. Fernandez-Fernandez B, Montoya-Ferrer A, Sanz AB, Sanchez-Niño MD, Izquierdo MC, Poveda J, et al. Tenofovir nephrotoxicity: 2011 update. AIDS Res Treat. 2011;2011:354908.

5. Canale D, Bragança AC, Gonçalves JG, Shimizu MH, Sanches TR, Andrade L, et al. Vitamin D deficiency aggravates nephrotoxicity, hypertension and dyslipidemia caused by tenofovir: role of oxidative stress and renin-angiotensin system. PLoS One. 2014;9:e103055.

6. Tourret J, Deray G, Isnard-Bagnis C. Tenofovir effect on the kidneys of HIV-infected patients: a double-edged sword? J Am Soc Nephrol. 2013;24:1519-27.

7. Libório AB, Andrade L, Pereira LV, Sanches TR, Shimizu MH, Seguro AC. Rosiglitazone reverses tenofovir-induced nephrotoxicity. Kidney Int. 2008;74:910-8.

8. Goicoechea M, Liu S, Best B, Sun S, Jain S, Kemper C, et al. Greater tenofovir-associated renal function decline with protease inhibitor-based versus nonnucleoside reverse-transcriptase inhibitor-based therapy. J Infect Dis. 2008;197:102-8.

9. Hill A, Hughes SL, Gotham D, Pozniak AL. Tenofovir alafenamide versus tenofovir disoproxil fumarate: is there a true difference in efficacy and safety? J Virus Erad. 2018;4:72-9.

10. Liu SN, Desta Z, Gufford BT. Probenecid-boosted tenofovir: a physiologically-based pharmacokinetic model-informed strategy for on-demand HIV preexposure prophylaxis. CPT Pharmacometrics Syst Pharmacol. 2020;9:40-7.
11. Wassner C, Bradley N, Lee Y. A review and clinical understanding of tenofovir: tenofovir disoproxil fumarate versus tenofovir alafenamide. J Int Assoc Provid AIDS Care. 2020;19:2325958220919231.

12. Tao X, Lu Y, Zhou Y, Zhang L, Chen Y. Efficacy and safety of the regimens containing tenofovir alafenamide versus tenofovir disoproxil fumarate in fixed-dose single-tablet regimens for initial treatment of HIV-1 infection: a meta-analysis of randomized controlled trials. Int J Infect Dis. 2020;93:108-17.

13. Pilkington V, Hughes SL, Pepperrell T, McCann K, Gotham D, Pozniak AL, et al. Tenofovir alafenamide vs. tenofovir disoproxil fumarate: an updated meta-analysis of 14894 patients across 14 trials. AIDS. 2020;34:2259-68.

14. Fraga RS, Van Vaisberg V, Mendes LC, Carrilho FJ, Ono SK. Adverse events of nucleos(t)ide analogues for chronic hepatitis B: a systematic review. J Gastroenterol. 2020;55:496-514.

15. Cotter AG, Sabin CA, Simelane S, Macken A, Kavanagh E, Brady JJ, et al. Relative contribution of HIV infection, demographics and body mass index to bone mineral density. AIDS. 2014;28:2051-60.

16. Kruger MJ, Nell TA. Bone mineral density in people living with HIV: a narrative review of the literature. AIDS Res Ther. 2017; $14: 35$

17. Conesa-Buendía FM, Llamas-Granda P, Larrañaga-Vera A, Wilder T, Largo R, Herrero-Beaumont G, et al. Tenofovir causes bone loss via decreased bone formation and increased bone resorption, which can be counteracted by dipyridamole in mice. J Bone Miner Res. 2019;34:923-38.

18. Havens PL, Kiser JJ, Stephensen CB, Hazra R, Flynn PM, Wilson CM, et al. Association of higher plasma vitamin D binding protein and lower free calcitriol levels with tenofovir disoproxil fumarate use and plasma and intracellular tenofovir pharmacokinetics: cause of a functional vitamin D deficiency? Antimicrob Agents Chemother. 2013;57:5619-28.

19. Casado JL. Renal and bone toxicity with the use of tenofovir: understanding at the end. AIDS Rev. 2016;18:59-68.

20. Freitas TQ, Franco AS, Bulhões CN, Pereira RM. Bone impairment in HIV-infected patients and tenofovir-induced osteomalacia as a differential diagnosis. Rev Med (Sao Paulo). 2018;97:372-3. 